\title{
cosmetics
}

ISSN 2079-9284

www.mdpi.com/journal/cosmetics

Review

\section{Relevance of Natural Phenolics from Grape and Derivative Products in the Formulation of Cosmetics}

\section{María Luisa Soto ${ }^{1,2}$, Elena Falqué ${ }^{3}$ and Herminia Domínguez ${ }^{1, *}$}

1 Departamento de Enxeñería Química, Universidade de Vigo (Campus Ourense), Edificio Politécnico, As Lagoas, Ourense 32004, Spain

2 Departamento de Imaxe Persoal, IES Lauro Olmo, O Barco de Valdeorras, Ourense 32300, Spain; E-Mail: sotoalvarez@edu.xunta.es

3 Departamento de Química Analítica, Universidade de Vigo (Campus Ourense), Edificio Politécnico, As Lagoas, Ourense 32004, Spain; E-Mail: efalque@uvigo.es

* Author to whom correspondence should be addressed; E-Mail: herminia@uvigo.es; Tel.: +34-988-387-082; Fax: +34-988-387-001.

Academic Editor: Martina Meinke

Received: 30 June 2015 / Accepted: 28 July 2015 / Published: 13 August 2015

\begin{abstract}
The consumer demand for natural ingredients in cosmetic products is increasing. Phenolic compounds are among the most studied natural antioxidant compounds, they also present antimicrobial, anti-inflammatory or antiaging actions and can permeate through the skin barrier. Grapes contain valuable phenolic components and grape byproducts are widely available low cost raw materials. This review presents an overview of the application of phenolic compounds from grape products and byproducts as sources of natural ingredients for cosmetics.
\end{abstract}

Keywords: grape; by-products; phenolic compounds; bioactivities; cosmetics

\section{Introduction}

Plant active compounds are gaining increased popularity as cosmetic ingredients since they can protect and cure the skin [1]. Compared with synthetic cosmetic products, herbal products are mild and biodegradable [2] and have biological and therapeutic activities. The use of cosmeceuticals, with the 
advantagees of cosmetics and pharmaceuticals products, has drastically risen in recent years for skin care, for improving skin appearance, and for treating several dermatologic conditions [3].

Phenolic compounds are secondary plant metabolites, produced in response to different stresses such as infections, wounding, ultraviolet (UV) irradiation, ozone, pollutants, etc. The flavonoids are the most abundant and biologically active phytonutrients, which can reduce the inflammation, inhibit tumor growth, have proapoptotic and anti-angiogenic actions, antimicrobial, antiviral, and antiaging properties, modulate the immune system, increase capillary resistance, protect the cardiovascular and neurological systems, limit weight gain, promote wound healing, etc. Polyphenolics are used in numerous sectors of the food and cosmetic industry as natural additives (natural coloring agents, conservative agents, natural antioxidants, nutritional additives) [4].

Grape (Vitis vinifera), one of the most commonly consumed fruits in the world, contains a variety of active compounds, including organic acids, oils and polyphenols. Melatonin ( $N$-acetyl-5-methoxytryptamine) is also present in grapes [5]. Grape is one of the richest fruit sources of polyphenols; simple phenolics are derivatives of hydroxycinnamic acid and hydroxybenzoic acid and polyphenols include flavonoids, stilbenes and proanthocyanidins. The stilbene resveratrol and its derivatives have received attention from scientists and have found increasing applications [6,7].

Traditionally, wine making by-products, which account for $20 \%-30 \%(w / w)$ of the total grapes used for wine elaboration, are mainly destined to the formulation of soil fertilizers and as substrate for biomass production and livestock feeds, and more than $70 \%$ of grape polyphenols remain in the pomace. Moreover, grape seeds may be separated from the pomace and used either for production of grape seed oil or as individual food supplements in the form of grape seed powder or grape seed extracts. Bioactive compounds from winery by-products have health promoting activities both in vitro and in vivo. Wine pomace is an interesting source for natural antioxidants with application in pharmacological, cosmetic, and food industries [8,9].

This work reviews the major phenolic bioactives in grape products and byproducts, their cosmetic activities and the products in which they can be incorporated.

\section{Phenolic Composition}

\subsection{Chemical Classification of Phenolic Compounds}

Plants produce many different secondary metabolites with bioactive properties, and phenolic compounds are an example of these compounds. They are found predominantly in fruits, vegetables, herbs, roots, leaves, and seeds and they play an important physiological and morphological role in many functions, such as structure, innate defence system, reproduction or sensorial properties (colour, bitterness, taste, astringency and flavour) [10]. The level in plant sources depends on some factors such as cultivation techniques, cultivar, growing conditions, ripening process, processing and storage conditions, among others [11]. Their content may increase under stress conditions such as UV radiation, infection by pathogens and parasites, wounding, air pollution and exposure to extreme temperatures [12]. 
Phenolic compounds are formed through two metabolic pathways: the shikimic acid pathway where, mainly, phenylpropanoids are formed and the acetic acid pathway, in which the main products is the simple phenol [13]. Phenolic compounds comprise one or some aromatic rings and have one or more hydroxyl substituents. They are usually found as esters, methyl esters or glycosides rather than as free compounds, and mostly are present as conjugates with mono- and polysaccharides, linked to one or more of the phenolic groups [14].

The term "phenolics" covers a very large and diverse group of chemical compounds with heterogeneous structures, and can be classified in different ways [15]. The classification and some examples of the main phenolic compounds are presented in Figure 1.

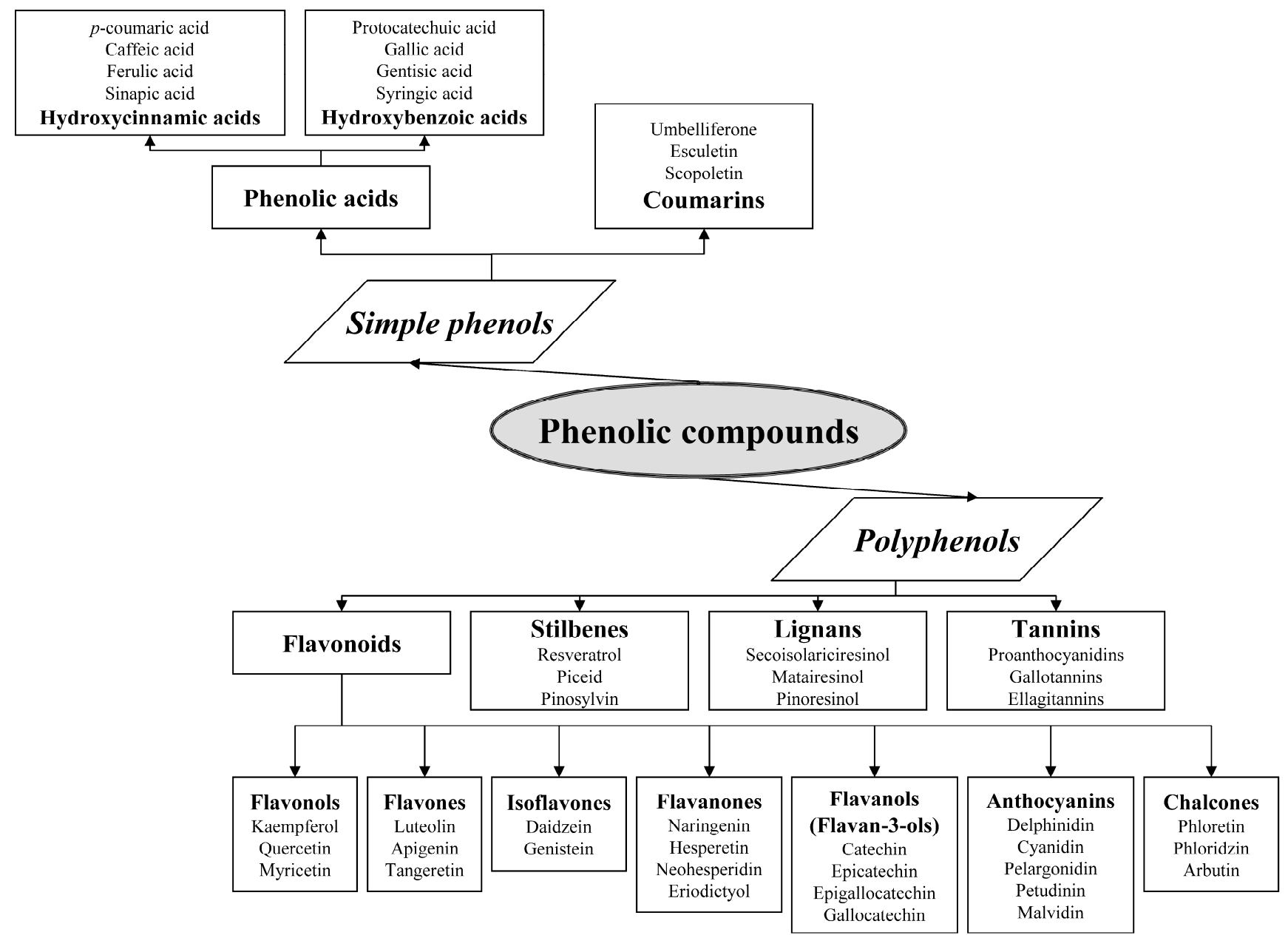

Figure 1. Classification of phenolic compounds.

The class and basic chemical structure of the phenolic compounds are presented in Table 1. 
Table 1. Chemical structures of the main phenolic compounds.

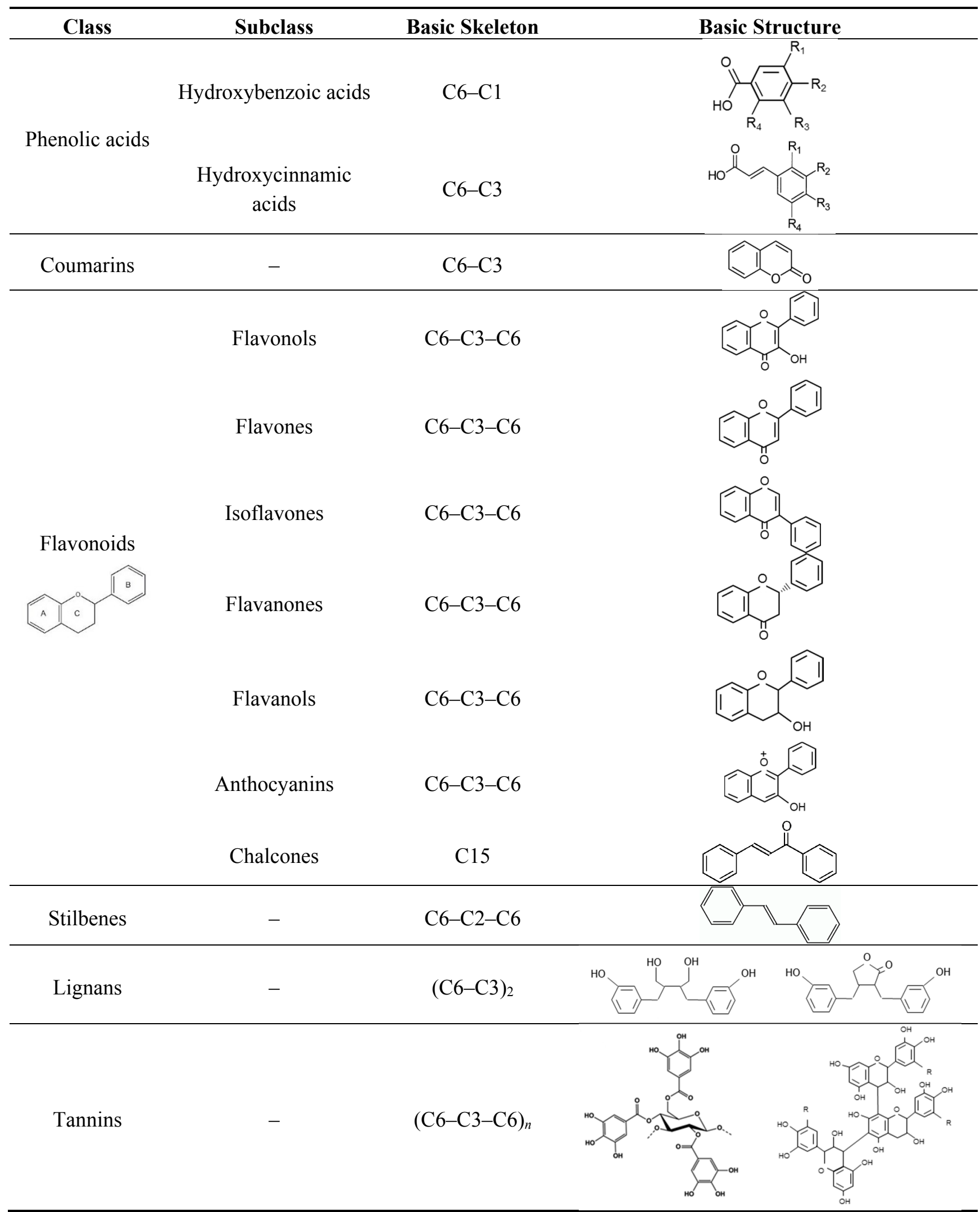

1. Phenolic acids are phenols with one carboxylic acid group and may be present in plants in free and bound forms. Phenolic acids are divided into two subgroups, the hydroxybenzoic and hydroxycinnamic acids and derivatives thereof. Hydroxybenzoic acids have in common the 
C6-C1 structure, and the hydroxycinnamic acids have aromatic compounds with a three-carbon side chain (C6-C3). The different phenolic acids differ in the number and position of the hydroxyl and methoxyl groups attached to the aromatic ring.

2. Coumarins, or chromones, are a class of secondary metabolites of plants derivatives from cinnamic acid by cyclization of the side chain of the $o$-coumaric acid [16]. Commonly, they are present as glycosides.

3. Flavonoids are the largest group of plant phenols and the most studied. The structure consists of 15 carbon atoms (C6-C3-C6). The aromatic ring $\mathrm{A}$ is derived from the acetate/malonate pathway, and ring $\mathrm{B}$ is derived from phenylalanine through the shikimate pathway [17]. Variations in substitution patterns to ring $\mathrm{C}$ (oxygenation, alkylation, glycosylation, acylation or sulfation) result in 13 flavonoid classes, being the most important flavonols, flavones, isoflavones, flavanones, flavanols (also called flavan-3-ols) and anthocyanidins or anthocyanins [18]. The chalcones are intermediate in the biosynthesis of flavonoids.

4. Stilbenes are a small group of phenylpropanoids characterized by a 1,2-diphenylethylene backbone. Most plant stilbenes are derivatives of the basic unit trans-resveratrol (3,5,4'-trihydroxy-trans-stilbene). In plants that naturally produce stilbenes, these metabolites are generally accumulated in both free and glycosylated forms [19].

5. Lignans are formed of two phenylpropane units, which are commonly present in fruits, seeds, grains, trees and vegetables. Secoisolariciresinol and matairesinol were the first plant lignans identified, and later pinoresinol, lariciresinol and others [20].

6. Tannins are phenolic compounds of molecular weight between 500 and $3000 \mathrm{D}$ and may be subdivided into: hydrolysable, esters of gallic acid (gallo- and ellagitannins), condensed tannins (also known as proanthocyanidins), polymers of polyhydroxyflavan-3-ol monomers, and phlorotannins, found in brown seaweeds.

Plant extracts that are rich in antioxidants components will have high potential to be developed into cosmetic formulation [21]. Phenolic compounds form one of the main classes of secondary plant metabolites and several thousand (among them over 8000 flavonoids) different compounds have been identified with a large range of structures: monomeric, dimeric and polymeric phenols.

\subsection{Phenolic Compounds in Grapes Products and Byproducts}

Grapes (Vitis vinifera L.) are an abundant worldwide source of phenolic compounds, which are mostly found in skins and seeds. Most phenolics in wine come from the grape and the final values are influenced by winemaking process.

Flavonoids or proanthocyanidins are the most abundant in skins and seeds, in berries predominate the condensed tannins or polymers of flavan-3-ol, followed by the anthocyanins, also located in the skin [22]. Flavonols are found as free form in wines or bond (as glucosides, galactosides, quercetin-3-rutinoside- or rutin- and glucuronides) in grapes. Among phenolic acids, hydroxybenzoic and hydroxycinnamic acids, either in the free or conjugated forms are predominant. The most abundant and well-known stilbene is trans-resveratrol. 


\section{Activities}

\subsection{Ultraviolet Radiation Protection}

The increased levels of ultraviolet radiation reaching the earth surface and skin overexposure have contributed to a growing number of skin-related disorders. Depending on the wavelength UV radiation is classified into UVA (320-400 nm), UVB (280-320 nm) and UVC (200-280 nm). UVA radiation (typically $>90 \%$ of the total UV radiation reaching the earth surface) can penetrate deeper into the epidermis and dermis, induces the generation of reactive oxygen species (ROS), and after chronic exposure can cause premature photoaging of the skin. UVB radiation ( $4 \%-5 \%$ of the total UV radiation) can penetrate into the epidermis layer and induces adverse biological effects. UVC radiation is completely absorbed by atmospheric oxygen and ozone.

The harmful effects of UV radiation include erythema, edema, hyperpigmentation, photoaging, and skin cancer [23]. Melanin can absorb UV rays and protects skin cells from the detrimental effects of UV exposure. If the amount of melanin produced is not sufficient, sunburn can occur. In order to protect skin against UV radiation topical application of UV absorbing or reflecting active molecules has been proposed.

Nowadays, there is a worldwide trend to develop highly effective UV protection chemical sunscreens, since the incidence of skin cancers have increased over the past few decades. Naturally occurring compounds have gained considerable attention since most show antioxidant, anti-inflammatory, and immunomodulatory properties, which provide further protection against the damaging effects of UV radiation exposure. However, the optimal dose of active constituents in natural extracts, compatibility, concentration, and stability should be established. The use of natural compounds in combination with synthetic agents may provide an effective strategy.

Sunscreens can be inorganic, inert particles reflecting the radiations without causing allergic sensitization, but have a cosmetically undesirable visual effect, and organic sunscreens, generally conjugated aromatic compounds, with more cosmetic appeal, may be activated by UV radiation and produce photosensitizer molecules causing adverse skin reactions. Different substances used in cosmeceuticals formulations could be useful for individuals who have had previous skin cancers and need to prevent possible new lesions [24].

Phenolic acids and flavonoids are efficient protectors through reduction of oxidative stress, inflammation, and immunosuppression and may be important component in cosmetic formulations for post-sun skin care $[25,26]$. Their topical application is efficient to inhibit erythema. There is increasing interest in systemic and topical applications of plant polyphenols for sun protection of skin. Among secondary metabolites acting as UV blockers are phenolic acids, flavonoids, terpenoids, and mycosporine-like amino acids. Grapes and derived products and byproducts are among the natural sources with potential photoprotection properties against UV radiation, and have been considered for herbal cosmetic formulations [1,23]. Topical application of resveratrol to SKH-1 hairless mice before exposure to UVB radiation resulted in significant inhibitions of UVB-mediated skin edema, inflammation and lipid peroxidation in the skin [23]. The stilbenoid resveratrol and the flavonoid quercetin attenuate responses of epidermal keratinocytes exposed to physiologically relevant dose of solar-simulated UV irradiation [26]. 


\subsection{Antioxidant and Antiaging}

Antioxidants may neutralize oxidative stress by inhibiting free radical formation, interrupting autoxidation chain reactions, up-regulating and protecting cellular antioxidant defenses mechanisms, neutralizing the action of metal pro-oxidant ions, inhibiting the action of pro-oxidative enzymes and increasing the activities of other antioxidants. Flavonoids represent a large group of low molecular weight compounds with high antioxidant properties and their chemical structure allows them to reduce oxidative stress through numerous mechanisms.

The antioxidant properties of grape parts, products and byproducts is well known, including their chelating activities, high radical scavenging properties in relation to Trolox and Vitamins C and E [27] and the potential to inhibit lipid oxidation in various food and cell models [8,27,28]. The consumption of grape-derived dietary flavonoids in the form of grape extracts and grape seed powders has been shown to effectively suppress oxidative stress and prevent oxidative damage in vivo [29]. However, phenolic compounds can have deleterious effects on the skin, since they can be unstable and led to the formation of radicals, or behave as prooxidants [30]. When they act as antioxidants, they can also form radicals, having effects on the skin not yet satisfyingly answered. Further, they can form degradation products whose toxicological relevance is also not yet proven.

Ultraviolet light produces reactive oxygen species in skin, which accelerate aging by damaging DNA, proteins, lipids, and other cellular constituents. Skin aging is a complex, progressive deterioration caused by intrinsic and extrinsic or environmental factors. The skin is the largest body organ, its ability to self-repair with advanced age is limited due to telomere attrition, hormone exhaustion, oxidative stress, genetic events and ultraviolet radiation exposure, genomic instability and epigenetic mutations. The formation of free radicals, which can react with DNA, proteins, and fatty acids, can cause oxidative damage and is a widely accepted mechanism causing skin ageing, by inducing wrinkling, photoaging, elastosis, drying, roughness, appearance of fine lines, lack of elasticity, and de-or hyperpigmentation marks [1].

A Vitis vinifera shoot extract showed in vitro antioxidant properties when added to cultured normal human keratinocytes and showed stronger antioxidant activity than Vitamin $\mathrm{E}$ and Vitamin $\mathrm{C}$. In in vivo studies' fluorimetric analysis reflected a decrease in the level of ROS in relation to controls and a dermatologic evaluation after four-week application showed improvements in the main clinical signs of photoaged skin [31].

Skin has a complex endogenous antioxidant system that protects it from oxidative damage, including nonenzymatic (ascorbic acid, tocopherol, ubiquinol, and glutathione) and enzymatic antioxidants (catalase, superoxide dismutase, thioredoxin reductase, glutathione peroxidase, and glutathione reductase) to maintain a balance between prooxidant and antioxidant. However, the natural antioxidant pool can be compromised by the oxidative stress caused by a variety of sources, including UV radiation and pollutants. The presence of excess free radicals leads to loss of cellular integrity, due to modification of DNA and abnormal expression of cellular genes, inducing increase in matrix metalloproteinases, which is responsible for the extracellular matrix protein degradation, causing wrinkle formation and metastases, as well as peroxidation of fatty acids within the phospholipid structure of the cell membrane [23].

The evaluation of the changes in epidermal structure and functionality over the lifetime and the identification of molecular mechanisms of epidermal signaling, maintenance, and differentiation, pointed to a need for new therapeutic alternatives to treat, prevent and recover skin aging. Targeting the 
molecular systems in skin shows potential for controlling skin aging [26]. To slow down skin aging when endogenous antioxidants become depleted and are insufficient, topical application or dietary ingestion of antioxidants is used [26].

Antiaging creams are predominantly moisturizer based skin care products. Topical antiaging products formulated with natural components make extensive claims to reduce wrinkles, fine lines, and sun damage, among others, and their sales are expected to increase [32], based on the consumer interest in health and appearance [33]. Topical antiaging products are complex formulations of cosmeceuticals, and enhance the growth of this segment of the market due to the demand of the population opting for less invasive, non-surgical alternatives to slow the effects of aging on the skin. Regarding the new active substance proposed to fight against skin-aging, the actual trends in cosmetics have two opposite values: a return to the past and a new beginning, with the utilization of traditional ingredients and the benefits from modern research and clinically tested products [34].

Natural polyphenols possess scavenging properties towards radical oxygen species that make them interesting for antiaging purposes in cosmetics or for nutraceutical applications. Grape provides active ingredients capable of counteracting symptoms of epidermal aging, such as phenolic components, which can improve solar radiation protection and epidermal antioxidant activity [35]. Grape-based creams are efficient in improving premature aging [36] and could reduce the occurrence of skin cancer and delay the process of photoaging [1]. Grape therapy has been proposed for the treatment of extrinsic skin aging processes [37,38]. Some commercial products contain pure natural compounds from grape, such as procyanidins, quercetin, or resveratrol [39], and melatonin [5], which are effective in delaying the onset of a variety of age-related diseases [40]. New phytoproducts for skin rejuvenation are in high demand, and in a study assessing the top currently marketed anti-aging creams, grape seed extract was found one of the most common botanicals [41].

A novel dietary supplement that contained grape seed extracts among other components (soy extract, fish protein polysaccharides, extracts from white tea, and tomato, Vitamins $\mathrm{C}$ and $\mathrm{E}, \mathrm{Zn}$ and chamomile extract) confirmed the benefits on healthy post-menopausal females compared to placebo for improving face condition, structure and firmness [42]. The presence of strong UV protectors and antiaging ingredients in a product rich in anthocyanins, obtained from grape cell suspension of $V$. vinifera L. may have beneficial effects on skin [29]. Quercetin protects human keratinocytes from UVA damage mainly by elevating intracellular antioxidant potential [43]. In cosmetics, stem cells extracted from substances of vegetable origin, rich in polyphenols such as resveratrol, and luteolin or peptides able to activate the genes of the Nicotinamide adenine dinucleotide NAD-dependent sirtuins [44]. A $V$. vinifera shoot extract had significantly stronger in vitro antioxidant capacity than Vitamin $\mathrm{C}$ or Vitamin $\mathrm{E}$ on keratinocytes and topically applied in a serum improved the main clinical signs of photoaged skin [5]. Furthermore, resveratrol and flavonoids are active substances in dermatological formulations for the prevention, slowing, or reversion of skin tumorigenesis $[45,46]$.

\subsection{Depigmenting}

Tyrosinase is responsible for coloring of skin, hair and eyes in animals. The addition of skin-lightening agents to the cosmetic formulations has become increasingly popular. Common depigmenting ingredients include hydroquinone, ascorbic acid, kojic acid, and liquorice extract 
(glabridin). Resveratrol, an original substrate for tyrosinase with promising cosmetic perspectives, is biotransformed by tyrosinase into an oxydated form, which acts as a powerful inhibitor of tyrosinase and was proposed as an additive in whitening cosmetics, particularly with a Morus alba extract [47]. Oxyresveratrol showed 32-fold stronger inhibitory effect on mushroom tyrosinase activity than kojic acid. Other stilbene derivatives are also efficient agents and the number and position of hydroxy substituents seem to play an important role in the inhibitory effects of stilbene compounds on tyrosinase activity [48]. The cosmetic use of resveratrol and oxyresveratrol has been partly limited by their chemical instabilities. The acetylated derivatives triacetyl resveratrol and tetraacetyl oxyresveratrol are less susceptible to oxidative discoloration than resveratrol and oxyresveratrol, respectively, less active tyrosinase inhibitors and are as effective as the original compounds for cellular melanogenesis inhibition [49]. However, it is possible that topical application of resveratrol-containing products to skin exposed to sunlight could produce potential hazardous action [43]. Side effects need also to be considered with phytochemicals used for lightening epidermal melasma and UV radiation causing hyperpigmentation [50].

The skin pigmentation induced by UV was reduced by the oral administration of $1 \%$ of a grape seed extract (containing 89.3\% proanthocyanidins) one week after UV-induced pigmentation of guinea pig skin. The number of 3,4-dihydroxyphenylalanine (DOPA)-positive melanocytes, melanin 8-hydroxy-20-deoxyguanosine and melanin-Ki-67-positive cells, and melanin proliferating cell nuclear antigen-positive cells decreased in the grape seed extract group compared to the control group [5].

A stable water-in-oil (W/O) emulsion containing 2\% grape seed extract from a blue-black grape variety Muscat Hamburg improved the skin appearance, after application for eight weeks during a winter period in young adult and healthy volunteers on human cheek skin in comparison with the placebo [36].

\subsection{Anti-Inflammatory Action}

Inflammation is a protective response of tissues against cell injury, irritation, pathogen invasions, and serves as a mechanism for eliminating damaged and necrotic cells. Several environmental stress factors may cause inflammation. Under normal physiological conditions, a short period of acute inflammation can overcome negative effects on injured tissue. However, if inflammation is prolonged, chronic inflammation can develop and is considered to be a main mediator in the development of chronic diseases such as cancer, Alzheimer's, neurodegenerative diseases, cardiovascular diseases, diabetes, arthritis, and autoimmune and pulmonary diseases. Deregulation of precise control mechanism of inflammation leads to chronic inflammation and promotion of chronic disease. Grape polyphenols decrease chronic inflammation either by modulation of inflammatory pathways or by reducing ROS levels. Grape flavonoids and proanthocyanidins can target multiple pathways to overcome chronic inflammation, being more effective than some synthetic drugs. A freeze-dried extract of wine, which contains mainly flavonoids, anthocyanins, proanthocyanidins and hydroxycinnamic acid derivatives, showed higher anti-inflammatory activity when compared to indomethacin. Plant-derived phenylpropanoids can modulate different molecular pathways involved in the inflammatory responses in human cells triggered by different pro-inflammatory stimuli [51]. Proanthocyanidins in grape seeds have high anti-inflammatory action, because they scavenge free radicals, prevent lipid peroxidation and inhibit formation of pro-inflammatory cytokines. The suppression effect of extracts obtained by red and white grape pomaces on chronic inflammation induced by lipopolysaccharide and galactosamine, has 
been confirmed in several studies compiled by Georgiev et al. [29]. Wine phenolics may prevent or delay the progression of intestinal diseases characterized by oxidative stress and inflammation, especially because they reach higher concentrations in the gut than in other tissues [52].

\subsection{Wound Healing}

Non-healing wounds remain stagnant in the inflammatory stage, a mechanism during which phagocytic cells destroy invading pathogens and clear cellular debris. A by-product of this inflammation is the release from the neutrophils and macrophages of reactive oxygen species, which could induce cytokine and growth factor expression, but greatly increased ROS levels limit this beneficial effect in chronic wounds. A renewed interest in natural compounds with potential to promote angiogenesis and wound healing has arisen both for many chronic and vascular diseases and for cosmetic purposes. Among them, grape seed proanthocyanidin extract is shown to be efficient [53].

\subsection{Irritation}

Plant phenolics are active components of plants, traditionally used in medicine to treat chronic skin diseases when applied topically, i.e., to accelerate skin wound healing, exert anti-inflammatory effects, and protect human skin against deleterious effects of solar irradiation. Cosmetic/dermatological preparations containing phenolic extracts are sold with the claims of photoprotection, chemoprevention, anti-aging, wound healing, etc., but they could be a double-edged sword for human skin, exerting both protective and damaging actions. Polyphenols can be major contributors to adverse cutaneous reactions, namely skin irritation, inflammation, ulceration, allergic reactions, and/or irritant contact dermatitis, and can induce skin pigmentation defects. The molecular mechanisms of plant polyphenols/their metabolites interact with human skin components leading to opposite results: anti- and pro-oxidant properties, activation versus inhibition of the skin metabolic system, anti- and proinflammatory effects, and photoprotective and phototoxic actions have been discussed [43].

\subsection{Antimicrobial}

Ointments and cosmetic treatments containing grape/wine polyphenols have been used since ancient times for the treatment and prevention of some skin diseases [54]. Among the organic compounds produced by grapes as a defense against invading phytopathogens are numerous phenolic compounds, also active against human pathogens. Wines and winery byproducts and some of their bioactive components are efficient against foodborne, medical, and oral pathogenic bacteria [55]. Dose dependent antibacterial activities of the seed extracts were observed against some pathogenic and spoilage bacteria including Aeromonas hydrophila, Bacillus cereus, Enterobacter aerogenes, Enterococcus faecalis, Escherichia coli, E. coli O157:H7, Klebsiella pneumoniae, Mycobacterium smegmatis, Proteus vulgaris, Pseudomonas aeruginosa, Pseudomonas fluorescens, Salmonella enteritidis, S. typhimurium, Staphylococcus aureus and Yersinia enterocolitica [56]. Also, the extracts at $0.5 \%-1 \%$ had a bacteriostatic effect and at $2.5 \%-5 \%$ had a bactericidal effect against $E$. coli $\mathrm{O} 157: \mathrm{H} 7$ [56]. The inhibitory effects of grape seed extracts on biofilm formation are dose dependent and the effect is different against different bacteria. The lower efficiencies found at higher concentrations of the extract was caused by its poor 
dissolution in water [27]. Products derived from seed-free wine pomace showed bactericidal effects against total aerobic mesophilic bacteria and lactic acid bacteria and inhibited Enterobacteriaceae and the product derived from whole winery products presented bacteriostatic activity against these microorganisms [8]. Grapeseed extracts were active against Alicyclobacillus acidoterrestris vegetative cells and spores [57], and against Listeria monocytogenes [29]. Alcohol-free wine extracts and extracts from pomace, fermented seeds and skin have shown inhibitory action against Candida albicans. The antifungal activity of grape products made them attractive for being incorporated into skin care cosmetics, particularly those from grape seeds, which are active at lower concentrations, and may be useful and economically feasible as antibacterial agents [56].

The compounds causing oral malodour and the available oral care preparations used for treatments have been reviewed [58]. Excess of reactive oxygen species release is implicated in the inflammatory process, and the implication of oxidative stress in the pathogenesis of periodontitis was evidenced. Therefore, antioxidant properties in the prevention of periodontal diseases and for oral hygiene purposes should also be considered [27]. A grape seed extract was proposed for prevention or treatment of periodontal diseases, for its antibacterial effect on $F$. nucleatum and $P$. gingivalis, its antiplaque activity on a multispecies biofilm and its antioxidant capacity [27]. The inhibitory effect of phenolic compounds from grape seeds extracts is more potent against Gram-positive bacteria than against Gram-negative [59]. The ability of extracts of red grape seed to inhibit bacteria and plaque formation was confirmed [60]. Red wine and dealcoholized wine had an antimicrobial effect against $F$. nucleatum, S. oralis and A. oris [61].

\subsection{Antiobesity}

Wine components have been proposed as an alternative natural approach to prevent or treat inflammatory bowel diseases since they act as both free radical scavengers and modulators of specific inflammation-related genes, and have recently been considered for their ability to act as probiotics [52]. Grape flavonoids may play an important role in modulation of human gut microflora and thus could have beneficial effects in control of weight loss. Polyphenols in grapes and grape products may reduce metabolic syndrome and prevent development of obesity and type 2 diabetes, by acting as multi-target modulators with antioxidant and anti-inflammatory effects. Grape powder acutely improves glucose tolerance and grape seed extract prevents metabolic syndrome, type 2 diabetes and obesity, also by modulating of metabolic endotoxemia and improving of gut barrier integrity [29].

\section{Activities}

\subsection{Permeation}

A prerequisite for the action of grape compounds in human skin is their permeation through the skin barrier, in particular the stratum corneum. In vitro experiments of skin permeation confirmed the potential of polyphenols as antiaging active cosmetic ingredients, since mixtures of model phenolics (catechin, epigallocatechin gallate, resveratrol, quercetin, rutin and protocatechuic acid) formulated into $\mathrm{O} / \mathrm{W}$ emulsions could pass the stratum corneum barrier and were found mostly in the epidermis and dermis. The most permeable substance was protocatechuic acid, and the least permeable was quercetin [62]. 
Permeation of catechin and resveratrol into the porcine ear skin from the propylene glycol vehicle was evidenced. The amount of catechin in the stratum corneum was higher than the amount of resveratrol whereas in the underlying skin it was similar. Of the total amount of polyphenols in the skin, approximately $90 \%$ was retained in the stratum corneum whereas $10 \%$ was quantified in the underlying skin [63].

\subsection{Safety}

Vitis vinifera (grape) seed extract is reported to function as an oral care and anticaries, antidandruff, antifungal, antimicrobial, antioxidant, flavoring, light stabilizer, sunscreen and skin-conditioning agents. Pesticide residues and heavy metals that may be present in botanical ingredients should be controlled by the cosmetics industry to limit these impurities in the ingredient before blending into cosmetic formulation. Grape-derived ingredients did not produce systemic toxicity in oral single-dose or long-term (up to 12 months) repeated dose studies; grape color extract was not a reproductive or developmental toxicant; grape-derived ingredients do not appear to be irritants or sensitizers and an expert panel concluded the grape derived ingredients listed subsequently are safe in the present practices and concentrations in cosmetics [5].

A winery byproduct concentrate, containing more than $50 \%$ phenolics, useful for the formulation of cosmetic emulsions and oils, showed no irritation in an Episkin test [64,65]. Products containing $3 \%-10 \%$ grape extracts were nonirritant in a dermal irritation test in human skin [5]. Grape seed extract-based cream was safe, since similar effects on skin erythema and moisture contents and no hypersensitivity cases were found with Burchard test for placebo and cream and no skin hypersensitivity cases were observed [36]. However, some ocular irritation effects have been reported for grape seed extracts [5]. In addition, contradictory effects derived from the non-enzymatic and enzymatic polyphenol transformation in the skin, the possible prooxidant and proinflammatory actions and the photo-protective versus photo-toxic effects of these compounds should be considered [66].

\subsection{Encapsulation and Delivery}

Polyphenols present poor bioavailability mainly due to low water solubility and have limited stability towards light and heat and during processing, storage, and in the gastrointestinal tract, facts limiting their activity and the potential health benefits. The topical use of natural polyphenols is also delicate because of their significant sensitivity to environmental factors, including physical, chemical, and biological conditions.

Therefore, the administration of phenolic compounds requires the formulation of a finished protecting product able to maintain the structural integrity of the polyphenol until consumption or administration, mask its taste, increase its water solubility and bioavailability, and convey it towards a physiological target. Novel delivery systems have been developed, and among them encapsulation appears promising. Many encapsulation methods, physical, physicochemical, chemical methods, and other connected stabilization methods have been applied to plant polyphenols and are available for food, pharmaceutical, cosmetic and agricultural applications [4].

According to the needs related to a specific field of application, the encapsulated particles allow to: protect fragile or unstable compounds, protect the user from the side-effects of the encapsulated compound, keep their structure and improve their stability, control the release of the encapsulated compound, etc. A variety of encapsulation methods is available, including physical, physicochemical, 
chemical methods. The most commonly used encapsulation methods of natural polyphenols were reviewed. A mixture of maltodextrin and gum arabic has been used for encapsulation of grape seed procyanidins and epigallocatechin gallate. A sodium caseinate-soy lecithin matrix was used to encapsulate grape seed extract, apple extract and olive tree leaf extract, and the retention of the entrapped polyphenols preserved the antioxidant activity. Microparticles made of cross-linked grape proanthocyanidin were produced and the polyphenolic compound constitutes the membrane material, while the cross-linking reaction stabilizes the molecule and maintains the radical-scavenging activity [4].

The co-encapsulation of resveratrol/curcumin and alpha-tocopherol/curcumin in niosomes controls and enhances the in vitro percutaneous permeation of the antioxidants, and the antioxidants combinations resulted in a promoted synergistic ability to reduce free radicals [67]. The release of polyphenol compounds from the vegetable and seed oil extracts was higher when they were included in gel than in emulsion based cosmetic formulations [37].

The properties, aesthetic appeal, improved topical absorption, performance and sensory attributes of cosmetic products can be enhanced with the newly developed encapsulation approaches, such as liposomes, phytosomes, transferosomes, nanoemulsions, nanoparticles, microemulsions, nanocrystals, and cubosomes [2,33]. The cosmeto-textiles with an active substance incorporated into their structure are used in the cosmetics and pharmaceutical industries and can act as a reservoir system capable of progressively delivering the active substance to the skin. An in vitro percutaneous absorption though pig skin demonstrated the delivery of the resveratrol from the textile to the dermis, epidermis, and stratum corneum [68].

\subsection{Examples of Products}

Wine has been used traditionally for healing and beauty purposes. However, the term wine therapy is relatively recent. At the end of the nineties, Matilde Cathiard and Bertrand Thomas inaugurated the first spa, "Les Sources de Caudalie", specializing in what was called wine therapy, near Bordeaux, and also a new cosmetic trademark was launched, based on grape phenolics. Nowadays, similar treatments are offered worldwide in different spas, usually located close to vineyards and wine factories. Wine therapy is usually referred to as stetic treatments performed with grapes and vine and grape parts, seed oil, grape juice, grape pomace. In wine therapy, spa hydrothermal techniques and grape derived products are combined, and the continued use can provide a relaxing effect as well as an improved blood circulation and muscular tonification. A sequence could include a relaxing bath with grape pomace and essential oils, followed by an exfoliating session with ground grape seeds mixed with red vine cream, a massage with red grape seed oil and/or with grape derived components and a final shower [69,70].

A variety of grape-derived ingredients are commercially used in cosmetic formulations: grape and extracts from grape bud, flower, fruit, fruit powder, fruit water, juice, juice extract, leaf oil, water and wax, leaf/seed/skin, root, seed, seed powder, shoot, skin, skin powder, vine, vine sap, hydrolyzed grape fruit and grape skin. Vitis vinifera seed oil and hydrogenated grapeseed oil are safe for use in cosmetics. Some examples of the activity of the major components and the cosmetic uses are summarized in Table 2. The concentrations are relatively low, grape leaf extract is included at up to $3 \%$ in leave-on formulations (perfumes) and grape fruit extract and grape juice are included at up to $2 \%$ in rinse-off skin cleansing products and others at 0.4 to 0.5 and $>0.5 \mathrm{~g} / \mathrm{kg}$, respectively [5]. 
Table 2. Examples of biological activities of grapes and their cosmetic application.

\begin{tabular}{lll}
\hline Compounds & Activity & Cosmetic Use \\
\hline $\begin{array}{l}\text { Anthocyanins, gallic acid, catechin, epicatechin, } \\
\text { conjugated flavonoids, proanthocyanidins, } \\
\text { resveratrol, melanin }\end{array}$ & $\begin{array}{l}\text { Antioxidant and radical } \\
\text { scavenging } \\
\text { Stimulation of cell growth }\end{array}$ & $\begin{array}{l}\text { Antiaging } \\
\text { Reaffirming } \\
\text { Preservatives }\end{array}$ \\
$\begin{array}{l}\text { Ferulic acid, caffeic acid, gallic acid, } \\
\text { proanthocyanidins, resveratrol }\end{array}$ & Photoprotector & $\begin{array}{l}\text { Sun protectors } \\
\text { After-sun } \\
\text { Lip balm protectors }\end{array}$ \\
\hline & & Chemical and physical \\
Tartaric acid & Exfoliating & Anfoliating agent \\
& Depigmenting & Antibrowning \\
& pH regulation & pH corrector \\
\hline Oleic, linoleic and linolenic acids & Antioxidant & Antiaging \\
Vitamins C and E & Nutritive & Day and night creams \\
\hline
\end{tabular}

\section{Conclusions}

Grape contains a wide variety of compounds with demonstrated therapeutic or health promoting properties. Among them, flavonoids are the most abundant and widely studied, with biological properties, including antioxidant, anti-inflammatory, anti-cancer, antimicrobial, antiviral, cardioprotective, neuroprotective, and hepatoprotective activities. Consumer demand for natural cosmetic formulations is increasing, but also the sensory attributes should be optimized. Novel and innovative delivery systems will help in the development of new cosmetic products and cosmeceutical ingredients. The growing interest in the biological activities of grape and grape products favour their valorization as a source of bioactive phytochemicals of application in pharmaceutical, cosmetic and food industries, and constitute an efficient, profitable, and environment-friendly alternative for the generated residues. Caution is needed with regard to some controversial effects derived from the use of plant polyphenols in cosmetology.

\section{Acknowledgments}

The authors are grateful to Xunta de Galicia (Conecta Peme Program and INBIOMED program) for the financial support. Both projects were partially funded by the FEDER Program of the European Union ("Unha maneira de facer Europa").

\section{Author Contributions}

The authors have equally contributed.

\section{Conflicts of Interest}

The authors declare no conflict of interest. 


\section{References}

1. Saraf, S.; Kaur, C. Phytoconstituents as photoprotective novel cosmetic formulations. Pharmacogn. Rev. 2010, 4, 1-11.

2. Chanchal, D.; Swarnlata, S. Novel approaches in herbal cosmetics. J. Cosmet. Dermatol. 2008, 7, 89-95.

3. Abdullah, B.J.; Nasreen, R. Cosmeceuticals: A revolution in cosmetic market. Int. J. Pharm. Technol. 2012, 4, 3925-3942.

4. Munin, A.; Edwards-Lévy, F. Encapsulation of natural polyphenolic compounds: A review. Pharmaceutics 2011, 3, 793-829.

5. Fiume, M.M.; Bergfeld, W.F.; Belsito, D.V.; Hill, R.A.; Klaassen, C.D.; Liebler, D.C.; Marks, J.G.; Shank, R.C.; Slaga, T.J.; Snyder, P.W.; et al. Safety assessment of Vitis vinifera (Grape)-derived ingredients as used in cosmetics. Int. J. Toxicol. 2014, 33, 48-83.

6. Delmas, D. Resveratrol: Sources, Production and Health Benefits; Nova Science Publishers Inc.: Hauppauge, NY, USA, 2013.

7. Pezzuto, J.M.; Kondratyuk, T.P.; Ogas, T. Resveratrol derivatives: A patent review (2009-2012). Expert Opin. Ther. Pat. 2013, 23, 1529-1546.

8. García-Lomillo, J.; González-SanJosé, M.L.; del Pino-García, R.; Rivero-Pérez, M.D.; Muñiz-Rodríguez, P. Antioxidant and antimicrobial properties of wine byproducts and their potential uses in the food industry. J. Agric. Food Chem. 2014, 62, 12595-12602.

9. Teixeira, A.; Baenas, N.; Domínguez-Perles, R.; Barros, A.; Rosa, E.; Moreno, D.A.; García-Viguera, C. Natural bioactive compounds from winery by-products as health promoters: A review. Int. J. Mol. Sci. 2014, 15, 15638-15678.

10. Balasundram, N.; Sundram, K.; Samman, S. Phenolic compounds in plants and agri-industrial by-products: Antioxidant activity, occurrence, and potential uses. Food Chem. 2006, 99, 191-203.

11. Naczk, M.; Shahidi, F. Phenolics in cereals, fruits and vegetables: Occurrence, extraction and analysis. J. Pharm. Biomed. 2006, 41, 1523-1542.

12. Tomás-Barberán, F.A.; Espín, J.C. Phenolic compounds and related enzymes as determinants of quality in fruits and vegetables. J. Sci. Food Agric. 2001, 81, 853-876.

13. Bravo, L. Polyphenols: Chemistry, dietary sources, metabolism, and nutritional significance. Nutr. Rev. 1998, 56, 317-333.

14. Shahidi, F.; Naczk, M. Food Phenolics: Sources, Chemistry, Effects, Applications; Technomic Publishing Company Inc.: Lancaster, PA, USA, 1995.

15. Reis Giada, M.L. Food phenolic compounds: Main classes, sources and their antioxidant power. In Oxidative Stress and Chronic Degenerative Diseases-A Role for Antioxidants; Morales González, J.A., Ed.; InTech Publisher: Rijeka, Croatia, 2013; pp. 87-112.

16. Matern, V.; Lüer, P.; Kreusch, D. Biosynthesis of coumarins. In Comprehensive Natural Products Chemistry: Polyketides and other Secondary Metabolites Including Fatty Acids and Their Derivatives; Barton, D., Nakanishi, K., Meth-Cohn, O., Sankawa, U., Eds.; Pergamon Press: Oxford, UK, 1999; pp. 623-637.

17. Merken, H.M.; Beecher, G.R. Measurement of food flavonoids by high performance liquid chromatography: A review. J. Agric. Food Chem. 2000, 48, 577-599. 
18. Hollman, P.C.H.; Katan, M.B. Dietary flavonoids: Intake, health effects and bioavailability. Food Chem. Toxicol. 1999, 37, 937-942.

19. Chong, J.; Poutaraud, A.; Hugueney, P. Metabolism and roles of stilbenes in plants. Plant Sci. 2009, 117, 143-155.

20. Holmbom, B.; Eckerman, C.; Eklund, P.; Hemming, J.; Nisula, L.; Reunanen, M.; Sjöholm, R.; Sundberg, A.; Sundberg, K.; Willför, S. Knots in trees-A new rich source of lignans. Phytochem. Rev. 2003, 2, 331-340.

21. Sytar, O.; Brestic, M.; Rai, M.; Shao, H.B. Plant phenolic compounds for food, pharmaceutical and cosmetics production. J. Med. Plants Res. 2012, 6, 2526-2539.

22. Teissedre, P.L.; Chervin, C. Grape. In Health-Promoting Properties of Fruits and Vegetables; Terry, L.A., Ed.; CABI Press: Oxford, UK, 2011; Volume 487, pp. 154-170.

23. Saewan, N.; Jimtaisong, A. Natural products as photoprotection. J. Cosmet. Dermatol. 2015, 14, 47-63.

24. França, K.; Cohen, J.L.; Grunebaum, L. Cosmeceuticals for recurrence prevention after prior skin cancer: An overview. J. Drugs Dermatol. 2013, 12, 516-518.

25. Potapovich, A.I.; Kostyuk, V.A.; Kostyuk, T.V.; de Luca, C.; Korkina, L.G. Effects of pre- and post-treatment with plant polyphenols on human keratinocyte responses to solar UV. Inflammat. Res. 2013, 62, 773-780.

26. Peng, Y.; Xuan, M.; Leung, V.Y.L.; Cheng, B. Stem cells and aberrant signaling of molecular systems in skin aging. Ageing Res. Rev. 2015, 19, 8-21.

27. Furiga, A.; Lonvaud-Funel, A.; Badet, C. In vitro study of antioxidant capacity and antibacterial activity on oral anaerobes of a grape seed extract. Food Chem. 2009, 113, 1037-1040.

28. Zhou, K.; Raffoul, J.J. Potential anticancer properties of grape antioxidants. J. Oncol. 2012, 2012, $1-8$.

29. Georgiev, V.; Ananga, A.; Tsolova, V. Recent advances and uses of grape flavonoids as nutraceuticals. Nutrients 2014, 6, 391-415.

30. Kyselova, Z. Toxicological aspects of the use of phenolic compounds in disease prevention. Interdiscip. Toxicol. 2011, 4, 173-183.

31. Cornacchione, S.; Sadick, N.S.; Neveu, M.; Talbourdet, S.; Lazou, K.; Viron, C.; Renimel, I.; de Quéral, D.; Kurfurst, R.; Schnebert, S.; et al. In vivo skin antioxidant effect of a new combination based on a specific Vitis vinifera shoot extract and a biotechnological extract. J. Drugs Dermatol. 2007, 6, 8-13.

32. Nolan, K.A.; Marmur, E.S. Over-the-counter topical skincare products: A review of the literature. J. Drugs Dermatol. 2012, 11, 220-224.

33. Sharma, B.; Sharma, A. Future prospect of nanotechnology in development of anti-ageing formulations. Int. J. Pharm. Sci. 2012, 4, 57-66.

34. Cohen-Letessier, A. Cosmetics in skin aging. Ann. Dermatol. Venereol. 2009, 136, 367-371.

35. Lorencini, M.; Brohem, C.A.; Dieamant, G.C.; Zanchin, N.I.T.; Maibach, H.I. Active ingredients against human epidermal aging. Ageing Res. Rev. 2014, 15, 100-115.

36. Sharif, A.; Akhtar, N.; Khan, M.S.; Menaa, A.; Menaa, B.; Khan, B.A.; Menaa, F. Formulation and evaluation on human skin of a water-in-oil emulsion containing Muscat Hamburg black grape seed extract. Int. J. Cosmet. Sci. 2015, 37, 253-258. 
37. Sánchez, V.; Sandoval, C.; Franco, C. An evaluation of polyphenol release from cosmetic formulations. Ars. Pharm. 2008, 49, 309-320.

38. Ratz-Lyko, A.; Arct, J.; Majewski, S.; Pytkowska, K. Influence of polyphenols on the physiological processes in the skin. Phytother. Res. 2015, 29, 509-517.

39. Kusumawati, I.; Indrayanto, G. Natural antioxidants in cosmetics. Stud. Nat. Prod. Chem. 2013, 40, 485-505.

40. Bombardelli, E.; Morazzoni, P. Vitis vinifera L. Fitoterapia 1995, 66, 291-317.

41. Cronin, H.; Draelos, Z.D. Top 10 botanical ingredients in 2010 anti-aging creams. J. Cosmet. Dermatol. 2010, 9, 218-225.

42. Skovgaard, G.R.L.; Jensen, A.S.; Sigler, M.L. Effect of a novel dietary supplement on skin aging in post-menopausal women. Eur. J. Clin. Nutr. 2006, 60, 1201-1206.

43. Korkina, L.; de Luca, C.; Pastore, S. Plant polyphenols and human skin: Friends or foes. Ann. N. Y. Acad. Sci. 2012, 1259, 77-86.

44. Ávila, L.M.; Torres, C.; Ponce, L.F.; Baena, Y.; Aristizábal, F.A. Stem cells, corners stone of rejuvenescence. Clarifying concepts. Med. Cután. Iber. Lat. Am. 2012, 40, 3-10.

45. Korkina, L.G.; de Luca, C.; Kostyuk, V.A.; Pastore, S. Plant polyphenols and tumors: From mechanisms to therapies, prevention and protection against toxicity of anti-cancer treatments. Curr. Med. Chem. 2009, 16, 3943-3965.

46. Korkina, L.G.; Pastore, S.; Dellambra, E.; de Luca, C. New molecular and cellular targets for chemoprevention and treatment of skin tumors by plant polyphenols: A critical review. Curr. Med. Chem. 2013, 20, 852-868.

47. Bernard, P.; Berthon, J.Y. Resveratrol: An original mechanism on tyrosinase inhibition. Int. J. Cosmet. Sci. 2000, 22, 219-226.

48. Kim, Y.M.; Yun, J.; Lee, C.K.; Lee, H.; Min, K.R.; Kim, Y. Oxyresveratrol and hydroxystilbene compounds. Inhibitory effect on tyrosinase and mechanism of action. J. Biol. Chem. 2002, 277, 16340-16344.

49. Park, J.; Park, J.H.; Suh, H.J.; Lee, I.C.; Koh, J.; Boo, Y.C. Effects of resveratrol, oxyresveratrol, and their acetylated derivatives on cellular melanogenesis. Arch. Dermatol. Res. 2014, 306, 475-487.

50. Fisk, W.A.; Agbai, O.; Lev-Tov, H.A.; Sivamani, R.K. The use of botanically derived agents for hyperpigmentation: A systematic review. J. Am. Acad. Dermatol. 2014, 70, 352-365.

51. Korkina, L., Kostyuk, V.; de Luca, C.; Pastore, S. Plant phenylpropanoids as emerging anti-inflammatory agents. Mini Rev. Med. Chem. 2011, 11, 823-835.

52. Biasi, F.; Deiana, M.; Guina, T.; Gamba, P.; Leonarduzzi, G.; Poli, G. Wine consumption and intestinal redox homeostasis. Redox Biol. 2014, 2, 795-802.

53. Morgan, C.; Nigam, Y. Naturally derived factors and their role in the promotion of angiogenesis for the healing of chronic wounds. Angiogenesis 2013, 16, 493-502.

54. Fehér, J.; Lengyel, G.; Lugasi, A. The cultural history of wine-Theoretical background to wine therapy. Cent. Eur. J. Med. 2007, 2, 379-391.

55. Friedman, M. Antibacterial, antiviral, and antifungal properties of wines and winery byproducts in relation to their flavonoid content. J. Agric. Food Chem. 2014, 62, 6025-6042.

56. Baydar, N.G.; Sagdic, O.; Ozkan, G.; Cetin, S. Determination of antibacterial effects and total phenolic contents of grape (Vitis vinifera L.) seed extracts. Int. J. Food Sci. Technol. 2006, 41, 799-804. 
57. Molva, C.; Baysal, A.H. Antimicrobial activity of grape seed extract on Alicyclobacillus acidoterrestris DSM 3922 vegetative cells and spores in apple juice. LWT-Food Sci. Technol. 2015, 60, 238-245.

58. Lourith, N.; Kanlayavattanakul, M. Oral malodour and active ingredients for treatment. Int. J. Cosmet. Sci. 2010, 32, 321-329.

59. Delgado Adámez, J.; Gamero Samino, E.; Valdés Sánchez, E.; González-Gómez, D. In vitro estimation of the antibacterial activity and antioxidant capacity of aqueous extracts from grape-seeds (Vitis vinifera L.). Food Control 2012, 24, 136-141.

60. Smullen, J.; Finney, M.; Storey, D.M.; Foster, H.A. Prevention of artificial dental plaque formation in vitro by plant extracts. J. Appl. Microbiol. 2012, 113, 964-973.

61. Muñoz-González, I.; Thurnheer, T.; Bartolomé, B.; Moreno-Arribas, M.V. Red wine and oenological extracts display antimicrobial effects in an oral bacteria biofilm model. J. Agric. Food Chem. 2014, 62, 4731-4737.

62. Zillich, O.V.; Schweiggert-Weisz, U.; Hasenkopf, K.; Eisner, P.; Kerscher, M. Release and in vitro skin permeation of polyphenols from cosmetic emulsions. Int. J. Cosmet. Sci. 2013, 35, 491-501.

63. Abla, M.J.; Banga, A.K. Quantification of skin penetration of antioxidants of varying lipophilicity. Int. J. Cosmet. Sci. 2013, 35, 19-26.

64. Díaz-Reinoso, B.; Gomes, A.; Freitas, M.; Fernandes, E.; Nogueira, D.R.; González, J.; Moure, A.; Levoso, A.; Vinardell, M.P.; Mitjans, M.; et al. Valuable polyphenolic antioxidants from wine vinasses. Food Bioproc. Technol. 2012, 5, 2708-2716.

65. Balboa, E.M.; Soto, M.L.; Nogueira, D.R.; González-López, N.; Conde, E.; Moure, A.; Vinardell, M.P.; Mitjans, M.; Domínguez, H. Potential of antioxidant extracts produced by aqueous processing of renewable resources for the formulation of cosmetics. Ind. Crop. Prod. 2014, 58, 104-110.

66. Korkina, L.G.; Pastore, S.; de Luca, C.; Kostyuk, V.A. Metabolism of plant polyphenols in the skin: Beneficial versus deleterious effects. Curr. Drug. Metabolism. 2008, 9, 710-729.

67. Tavano, L.; Muzzalupo, R.; Picci, N.; de Cindio, B. Co-encapsulation of lipophilic antioxidants into niosomal carriers: Percutaneous permeation studies for cosmeceutical applications. Colloid Surf. B Biointerfaces 2014, 114, 144-149.

68. Alonso, C.; Martí, M.; Martínez, V.; Rubio, L.; Parra, J.L.; Coderch, L. Antioxidant cosmeto-textiles: Skin assessment. Eur. J. Pharm. Biopharm. 2013, 84, 192-199.

69. Armijo Valenzuela, M.; San Martín Bacaicoa, J. Curas balnearias y climáticas. Talasoterapia y Helioterapia. Available online: http://dspace.ceu.es/handle/10637/2351 (accessed on 15 July 2015). (In Spanish)

70. Carbajo, J.M.; Mestre J.; del Tío, R.; Ruiz Manso, R. Estética Hidrotermal; Videocinco: Madrid, Spain, 2007; pp. 181-186. (In Spanish)

(C) 2015 by the authors; licensee MDPI, Basel, Switzerland. This article is an open access article distributed under the terms and conditions of the Creative Commons Attribution license (http://creativecommons.org/licenses/by/4.0/). 